\title{
Health-related quality of life associated with trifluridine/tipiracil in heavily pretreated metastatic gastric cancer: results from TAGS
}

\author{
Josep Tabernero ${ }^{1}\left[\right.$ - Maria Alsina ${ }^{1} \cdot$ Kohei Shitara $^{2} \cdot$ Toshihiko Doi $^{2} \cdot$ Mikhail Dvorkin $^{3} \cdot$ Wasat Mansoor ${ }^{4}$. \\ Hendrik-Tobias Arkenau ${ }^{5} \cdot$ Aliaksandr Prokharau $^{6} \cdot$ Michele Ghidini $^{7}$. Catia Faustino ${ }^{8}$. Vera Gorbunova ${ }^{9}$. \\ Edvard Zhavrid $^{10} \cdot$ Kazuhiro Nishikawa $^{11} \cdot$ Takayuki Ando $^{12}$. Şuayib Yalçın ${ }^{13}$. Eric Van Cutsem ${ }^{14}$. Javier Sabater ${ }^{15}$. \\ Donia Skanji ${ }^{16}$. Catherine Leger ${ }^{16}$. Nadia Amellal ${ }^{16}$. David H. Ilson ${ }^{17}$
}

Received: 26 October 2019 / Accepted: 17 February 2020 / Published online: 4 March 2020

(c) The Author(s) 2020

\begin{abstract}
Background In TAGS, an international, double-blind, phase 3 trial, trifluridine/tipiracil significantly improved overall survival and progression-free survival compared with placebo in heavily pretreated metastatic gastric cancer patients. This paper reports pre-specified quality of life $(\mathrm{QoL})$ outcomes for TAGS.

Methods Patients were randomized 2:1 to trifluridine/tipiracil $\left(35 \mathrm{mg} / \mathrm{m}^{2}\right.$ twice daily on days $1-5$ and $8-12$ of each 28 -day cycle) plus best supportive care (BSC) or placebo plus BSC. QoL was evaluated at baseline and at each treatment cycle, using the EORTC QLQ-C30 and EORTC QLQ-STO22 questionnaires; results were considered valid for analysis only if $\geq 10 \%$ of patients completed the questionnaires. Key QoL outcomes were mean changes from baseline and time to deterioration in QoL. A post hoc analysis assessed the association between QoL and time to deterioration of Eastern Cooperative Oncology Group performance score (ECOG PS) to $\geq 2$.

Results Of 507 randomized patients, 496 had baseline QoL data available. The analysis cut-off was 6 cycles for trifluridine/ tipiracil and 3 cycles for placebo. In both treatment groups, there were no clinically significant deteriorations in the mean QLQ-C30 Global Health Status (GHS) score, or in most subscale scores. In a sensitivity analysis including death and disease progression as events, there was a trend towards trifluridine/tipiracil reducing the risk of deterioration of QoL scores compared with placebo. Deterioration in the GHS score was associated with deterioration in ECOG PS.

Conclusion QoL was maintained in TAGS, and there was a trend towards trifluridine/tipiracil reducing the risk of QoL deterioration compared with placebo.

Trial registration ClinicalTrials.gov number: NCT02500043
\end{abstract}

Keywords Gastric cancer $\cdot$ Health-related quality of life $\cdot$ Phase $3 \cdot$ Trifluridine/tipiracil

\section{Introduction}

Worldwide, gastric cancer is the fifth most common cancer and the third leading cause of cancer-related death [1]. The majority of patients present with advanced or metastatic disease and the prognosis for these patients is relatively poor [2], with a 5-year overall survival (OS) of less than 30\% [3]. After the failure of first- and second-line treatment, there are limited treatment options for patients with metastatic

Josep Tabernero

jtabernero@vhio.net

Extended author information available on the last page of the article gastric cancer. Thus, there is a need for effective agents with manageable safety profiles.

Trifluridine/tipiracil is an oral combination of the thymidine-based nucleoside analogue, trifluridine, and the thymidine phosphorylase inhibitor tipiracil hydrochloride [4, 5]. In TAGS, the randomized, double-blind, phase 3 trial in patients with heavily pretreated metastatic gastric cancer, trifluridine/tipiracil significantly improved median OS compared with placebo (5.7 vs 3.6 months), with a $31 \%$ reduction in risk of death (HR: 0.69 ; 2-sided $P=0.0006$ ) [6]. Trifluridine/tipiracil was also associated with significant improvements in progression-free survival (PFS; 2.0 vs 1.8 months; HR: $0.57 ; 2$-sided $P<0.0001)$ and time to Eastern Cooperative Oncology Group performance score 
(ECOG PS) deterioration to $\geq 2$ (31\% reduction in risk, HR: $0.69 ; 2$-sided $P=0.0005$ ) compared with placebo, and demonstrated a predictable and manageable safety profile [6]. Based on the results of TAGS, trifluridine/tipiracil was approved in the USA, the EU, and Japan for third-line treatment of metastatic gastric or gastroesophageal junction (GEJ) adenocarcinoma in adult patients [7-9].

Disease symptoms and drug toxicity can have a negative impact on patients' quality of life (QoL); therefore, in addition to OS, QoL is an important outcome to measure in trials in patients with cancer [10]. This is particularly true for patients with advanced cancer who may have a limited life expectancy, in which case any survival benefits must be weighed against treatment toxicity and impact on QoL [10]. Evaluation of QoL includes patient-reported physical, psychological and social dimensions, and best reflects how patients perceive their own state of health. In this paper, we report the effect of trifluridine/tipiracil versus placebo on patient-reported QoL, evaluated as a pre-specified endpoint in TAGS.

\section{Methods}

\section{Study design}

TAGS (ClinicalTrials.gov number: NCT02500043) was an international, randomized, double-blind, placebo-controlled, phase 3 trial in patients (aged $\geq 18$ years) with pre-treated ( $\geq 2$ regimens), histologically confirmed, non-resectable metastatic gastric adenocarcinoma, including adenocarcinoma of the gastroesophageal junction. Full study design details have been published previously [6]. Briefly, eligible patients were randomized 2:1 to receive either oral trifluridine/tipiracil $35 \mathrm{mg} / \mathrm{m}^{2}$ twice daily plus best supportive care (BSC) or placebo twice daily plus BSC on days 1-5 and 8-12 of each 28-day cycle. Previous regimens must have included a fluoropyrimidine, a platinum agent, and a taxane or irinotecan, or both. Patients whose tumors were HER2 positive must have received previous anti-HER2 therapy, if available. Randomization was stratified by region (Japan vs rest of World), ECOG PS (0 vs 1), and previous treatment with ramucirumab (yes vs no). Treatment continued until disease progression, unacceptable toxicity or patient withdrawal.

The study protocol was approved by the appropriate ethical review committees; the study was performed in accordance with the Declaration of Helsinki and Good Clinical Practice and all patients provided written, informed consent.

\section{QoL assessments}

QoL was a pre-specified secondary endpoint of TAGS and was evaluated using two validated, reliable and sensitive questionnaires [11]: the European Organization for Research and Treatment of Cancer (EORTC) Quality of Life Questionnaire-Core 30 (QLQ-C30) and the EORTC Quality of Life Questionnaire-Gastric Cancer Module (QLQ-STO22). EORTC QLQ-C30 was developed to assess the QoL of a wide range of cancer patients and incorporates a Global Health Status (GHS) scale, five functional scales (physical, role, cognitive, emotional and social), three symptom scales (fatigue, pain and nausea or vomiting) and six single items assessing additional symptoms commonly reported by cancer patients (dyspnea, loss of appetite, insomnia, constipation, diarrhea) and perceived financial impact of the disease [12]. The gastric cancerspecific questions on the EORTC QLQ-STO22 include four single-item subscales (dry mouth, body image, hair loss and problems with taste) and five multi-item subscales (dysphagia, dietary restriction, pain, upper gastroesophageal symptoms and emotional problems) [13]. This 22-item instrument was used alongside the QLQ-C30, resulting in a total of 52 items. A high score for a functional scale or global health item represents a better QoL (high level of functioning) whereas a high symptom score indicates a poorer QoL, i.e. a high level of symptoms.

QoL data were collected 1-7 days before randomization (baseline), prior to treatment administration on day 1 of each cycle (from cycle 2 onwards), and at the 30-day safety follow-up visit (if not performed in the past 4 weeks). The key pre-specified QoL outcomes were mean changes from baseline and time to deterioration in QoL. A post hoc analysis of the association between $\mathrm{QoL}$ and time to deterioration of ECOG PS to $\geq 2$ was also performed.

\section{Statistical analyses}

Analyses were conducted in all randomized patients who completed at least one baseline and post-baseline EORTC QLQ-C30 or QLQ-STO22 questionnaire. The compliance rate was calculated using the proportion of patients having completed a QoL questionnaire and the proportion of patients remaining in each cycle.

Mean changes in scores from baseline to each cycle were determined. Descriptive statistics for both multiitem scales and single-item measures were provided for each assessed time point. Statistics included number of patients, number of non-missing/missing scales, means with standard deviation (SD) and medians with range. For each time cycle, results were considered valid for analysis 
only if $\geq 10 \%$ of the intention to treat (ITT) patient population (all randomized patients) completed the questionnaires (calculated separately for each treatment arm). For both questionnaires, a mean change from baseline of at least 10 points was considered to be clinically relevant, while a change of at least 5 points was considered "a little change" [14].

For the median time to deterioration in QoL, 95\% confidence intervals (CI) were calculated; hazard ratios (HR) were calculated for between-group differences. Time to first deterioration in QoL was evaluated for each arm using Kaplan-Meier estimates and compared using the log-rank test. The main analysis of time to deterioration in QoL was defined as time to first deterioration by 5 points or more from baseline. Patients with no confirmed deterioration from baseline were censored at the time of their last observation. For this analysis, a Cox's proportional hazard model was used to adjust for baseline EORTC QLQ-C30 and QLQSTO22 scores, country and primary tumor type. Additionally, two sensitivity analyses were conducted. In the first, time to deterioration was defined as the first deterioration of $\geq 10$ points from baseline, and death was considered to be a deterioration event. The second sensitivity analysis also considered $\mathrm{a} \geq 10$-point deterioration and included death or progressive disease (PD) without previous deterioration in QoL as deteriorative events. These analyses used Cox proportional hazards models adjusting for the randomization stratification factors (region, ECOG PS at baseline, prior treatment with ramucirumab).

A post hoc exploratory analysis investigated the association between time to deterioration of ECOG PS to $\geq 2$ and changes in QoL throughout the study. To account for the longitudinally collected QoL scores, Cox proportional hazard models with time-dependent covariates were fitted to time-to-event data.

\section{Results}

\section{Baseline characteristics and questionnaire compliance}

The study was conducted in 110 academic hospitals in 17 countries. Between 24 February 2016 and 5 January 2018, 507 patients were enrolled and randomly assigned to receive trifluridine/tipiracil $(n=337)$ or placebo $(n=170)$. Baseline patient demographics and disease characteristics of the total patient population have been reported previously and were generally well balanced between the two groups [6]. Baseline QoL data was available for 332 (98.5\%) patients in the trifluridine/tipiracil group and 164 (96.5\%) patients in the placebo group.
Baseline compliance was similar between the two treatment groups; the number of patients completing the questionnaires decreased with each cycle, as the number of patients discontinuing treatment increased. The analysis cut-off point (i.e. including results only for time points at which $\geq 10 \%$ of the ITT population completed the questionnaires) was 6 cycles of treatment for trifluridine/tipiracil and 3 cycles for placebo (Table 1 ).

The mean baseline GHS, functioning and symptom scores were well balanced between the two treatment groups, with no differences of $>10$ points on either questionnaire (Table 2). For both trifluridine/tipiracil and placebo, the mean baseline QLQ-C30 GHS score was 58.4 (SD for trifluridine/tipiracil, 20.2; for placebo, 19.7). On the QLQ-C30 questionnaire, the most common symptoms were fatigue, pain and appetite loss, and on the QLQ-STO22, they were anxiety, body image and hair loss (Table 2).

\section{Change in QoL from baseline}

Although there was slight deterioration from baseline during treatment in both groups, there were no clinically relevant changes ( $\geq 10$ points) in the mean QLQ-C30 GHS scores (Fig. 1) or in most of the subscale scores at any time point for which there were sufficient data (Fig. 2). With trifluridine/tipiracil, there were no clinically relevant deteriorations in any of the subscale scores from baseline until end of cycle 6 , with the exception of deteriorations in the mean score of role functioning from baseline to the end of cycles 4 and $6(-10.2 \pm 24.2$ and $-13.4 \pm 30.0$, respectively). Clinically relevant deteriorations were seen in the placebo arm in the role functioning score from baseline to end of cycles 1 and

Table 1 Quality of Life questionnaire compliance rates for treatment cycles 1-6

\begin{tabular}{|c|c|c|c|c|}
\hline & \multicolumn{2}{|c|}{$\begin{array}{l}\text { Rate of patients with } \\
\text { QLQ-C } 30 \text { questionnaire } \\
\text { completed out of number of } \\
\text { patients in the cycle }\end{array}$} & \multicolumn{2}{|c|}{$\begin{array}{l}\text { Rate of patients with } \\
\text { QLQ-STO22 questionnaire } \\
\text { completed out of number of } \\
\text { patients in the cycle }\end{array}$} \\
\hline & $\begin{array}{l}\text { Trifluridine/tip- } \\
\text { iracil }(n=337)\end{array}$ & $\begin{array}{l}\text { Placebo } \\
(n=170)\end{array}$ & $\begin{array}{l}\text { Trifluridine/tip- } \\
\text { iracil }(n=337)\end{array}$ & $\begin{array}{l}\text { Placebo } \\
(n=170)\end{array}$ \\
\hline Baseline & 98.5 & 96.5 & 98.5 & 96.4 \\
\hline Cycle 1 & 84.2 & 76.8 & 84.1 & 76.8 \\
\hline Cycle 2 & 68.1 & 47.2 & 68.1 & 46.8 \\
\hline Cycle 3 & 85.5 & 69.7 & 85.3 & 69.7 \\
\hline Cycle 4 & 69.8 & 83.3 & 69.8 & 83.8 \\
\hline Cycle 5 & 86.2 & 66.7 & 86.2 & 66.7 \\
\hline Cycle 6 & 67.9 & 90.0 & 67.9 & 90.0 \\
\hline
\end{tabular}

EORTC, European Organization for Research and Treatment of Cancer; QLQ-C30, Quality of Life Questionnaire-Core 30; QLQ-STO22, Quality of Life Questionnaire-Gastric Cancer Module 
Table 2 Baseline quality of life scores

\begin{tabular}{|c|c|c|}
\hline & \multicolumn{2}{|c|}{ Baseline score, mean (SD) } \\
\hline & $\begin{array}{l}\text { Trifluridine/tipiracil } \\
\left(n=327-332^{\mathrm{a}}\right)\end{array}$ & $\begin{array}{l}\text { Placebo } \\
\left(n=162-164^{\mathrm{a}}\right)\end{array}$ \\
\hline \multicolumn{3}{|l|}{ QLQ-C30 } \\
\hline GHS & $58.4(20.23)$ & $58.4(19.72)$ \\
\hline \multicolumn{3}{|l|}{ Functioning scores $^{\mathrm{b}}$} \\
\hline Physical & $76.2(18.92)$ & $77.6(18.11)$ \\
\hline Role & $75.9(26.61)$ & $77.1(24.71)$ \\
\hline Emotional & $77.5(21.31)$ & $79.9(18.30)$ \\
\hline Cognitive & $85.1(18.75)$ & 86.7 (17.87) \\
\hline Social & $79.4(23.98)$ & $79.6(22.85)$ \\
\hline \multicolumn{3}{|l|}{ Symptom scores ${ }^{\mathrm{c}}$} \\
\hline Fatigue & $35.9(21.08)$ & $35.9(22.86)$ \\
\hline Nausea and vomiting & $11.6(18.93)$ & $10.9(19.01)$ \\
\hline Pain & $27.0(25.65)$ & $28.8(26.32)$ \\
\hline Dyspnea & $15.9(23.27)$ & $17.3(22.90)$ \\
\hline Insomnia & $24.4(27.94)$ & $22.3(27.98)$ \\
\hline Appetite loss & $27.8(29.24)$ & $26.6(29.58)$ \\
\hline Constipation & $14.6(22.73)$ & $18.4(26.23)$ \\
\hline Diarrhea & $13.2(22.56)$ & $9.0(18.91)$ \\
\hline Financial difficulties & $17.7(25.34)$ & $17.6(26.53)$ \\
\hline \multicolumn{3}{|l|}{ QLQ-STO22 } \\
\hline Dysphagia & $11.4(17.46)$ & $11.1(18.45)$ \\
\hline Dietary restrictions & $21.0(19.51)$ & $21.3(21.20)$ \\
\hline Pain & $23.3(20.79)$ & $22.9(20.75)$ \\
\hline Upper gastroesophageal & $14.4(18.04)$ & $14.9(17.82)$ \\
\hline Anxiety & $41.7(24.20)$ & $43.6(25.94)$ \\
\hline Dry mouth & $21.9(25.44)$ & $21.7(25.54)$ \\
\hline Body image & $26.4(29.65)$ & 30.7 (30.76) \\
\hline Hair loss & $26.8(31.75)$ & $23.3(27.19)$ \\
\hline Taste problems & $19.1(25.75)$ & $17.4(24.94)$ \\
\hline
\end{tabular}

GHS, Global Health Status; QLQ-C30, Quality of Life Questionnaire-Core 30; QLQ-STO22, Quality of Life Questionnaire-Gastric Cancer Module; SD, standard deviation

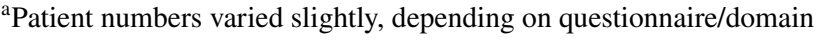
${ }^{\mathrm{b}} \mathrm{A}$ high function score represents a better $\mathrm{QoL}$

${ }^{\mathrm{c}} \mathrm{A}$ high symptom score represents a poorer QoL

2, fatigue and pain scores from baseline to end of cycle 2, and appetite loss from baseline to end of cycle 1 .

No clinically relevant differences ( $\geq 10$ points) between treatment groups over time were observed, with the following exceptions: the QLQ-C30 pain score at end of cycle 2 (change from baseline was 11.3 points higher for trifluridine/tipiracil than placebo) and the role functioning score at end of cycle 3 (the placebo score

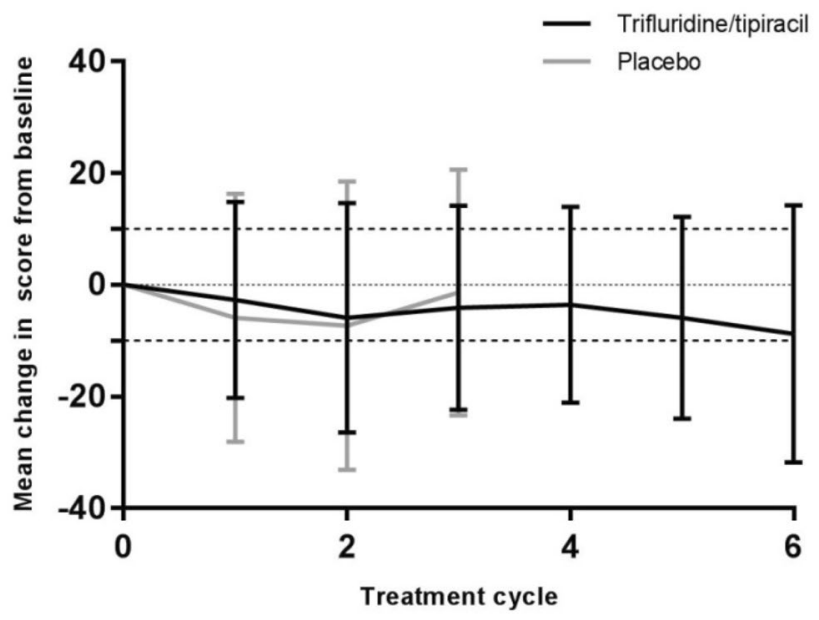

Fig. 1 Change from baseline across treatment cycles* in the EORTC QLQ-C30 GHS score EORTC, European Organization for Research and Treatment of Cancer; GHS, global health status; QLQ-C30, Quality of Life Questionnaire-Core 30.*Results were considered valid for analysis only if $\geq 10 \%$ of the original patient population completed the questionnaires; this corresponded to 3 cycles of treatment with placebo and 6 cycles for trifluridine/tipiracil. A high score represents a high quality of life. A mean change from baseline of $\geq 10$ points is considered clinically relevant

improved from baseline by 1.4 points and the trifluridine/ tipiracil score deteriorated from baseline by 8.6 points).

\section{Time to deterioration in QoL scores}

In the main analysis, the median time to deterioration by $\geq 5$ points in the QLQ-C30 GHS score was 2.6 months (95\% CI 2.3, 3.3) for trifluridine/tipiracil and 2.3 months (95\% CI 1.4-not estimable) for placebo (HR 1.27; 95\% CI 0.85-1.87).

In the sensitivity analysis including death as an event, the risk of deterioration (by $\geq 10$ points) in the QLQ-C30 GHS score was numerically lower with trifluridine/tipiracil than placebo (HR 0.92, 95\% CI 0.74-1.16); similar findings were observed for all QLQ-C30 and QLQ-STO22 scores, with the exception of the physical functioning score (Fig. 3a). In this analysis, the median time to deterioration (by $\geq 10$ points) in the QLQ-C30 GHS was 3.19 months (95\% CI 2.80-3.82) for trifluridine/tipiracil and 2.27 months (95\% CI 2.07-3.36) for placebo.

In the second sensitivity analysis which included death or PD as an event, compared with placebo, trifluridine/ tipiracil reduced the risk of deterioration (by $\geq 10$ points) for all QLQ-C30 and QLQ-STO22 scores (HRs ranged from 0.55 to 0.75 ; Fig. 3 b). For the QLQ-C30 GHS score, the median time to deterioration in this analysis was 


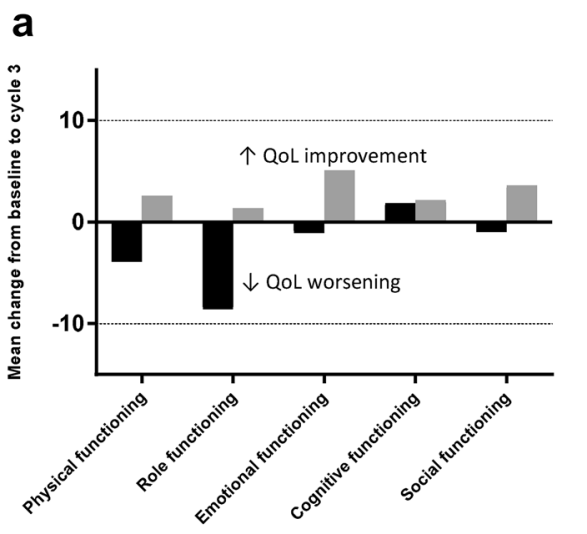

b

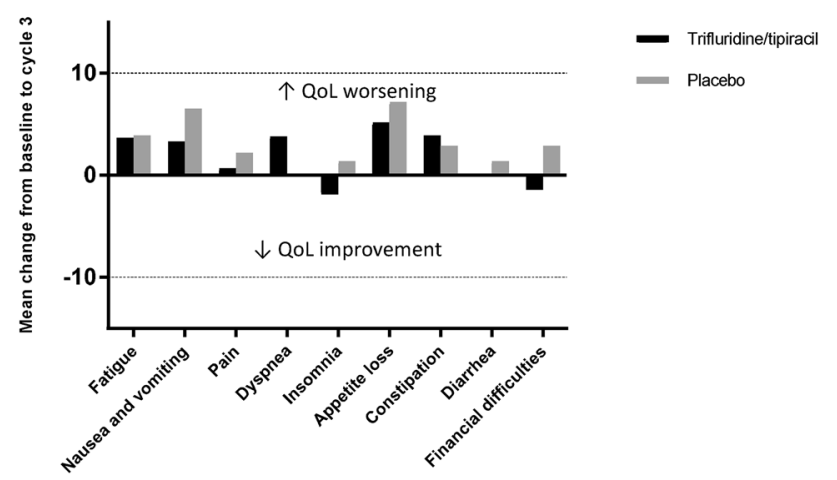

C

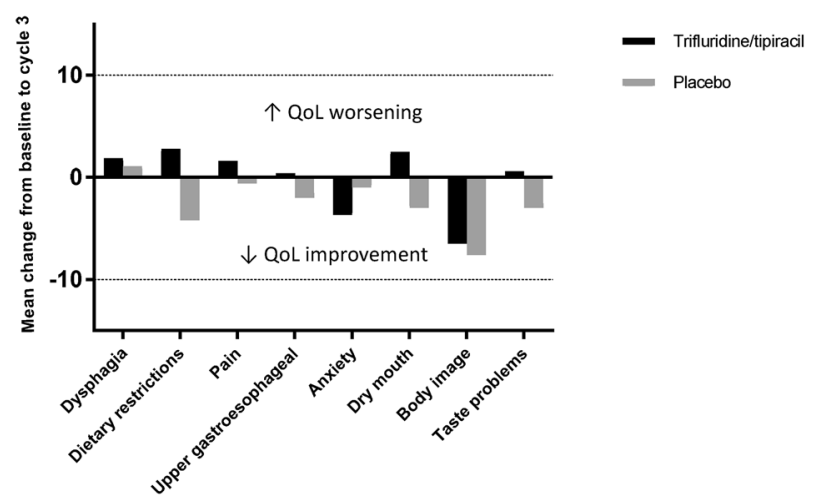

Fig. 2 Change from baseline to treatment cycle 3 in the EORTC (a) QLQ-C30 function scores (b) QLQ-C30 symptom scores and (c) QLQ-STO22 subscores. EORTC, European Organization for Research and Treatment of Cancer; GHS, Global Health Status; QLQ-C30, Quality of Life Questionnaire-Core 30; QLQ-STO22, Quality of Life Questionnaire-Gastric Cancer Module

2.11 months (95\% CI 2.07-2.27) for trifluridine/tipiracil versus 1.88 months (95\% CI 1.84-1.94) for placebo (HR $0.65,95 \%$ CI $0.52-0.81$; Fig. 3b).

\section{Association between QoL and time to ECOG deterioration}

Deteriorations in QoL scores of $\geq 10$ points were associated with a significantly increased risk of deterioration in ECOG PS to $\geq 2$ for the QLQ-C30 GHS score, all QLQ-C30 functional scale scores, the QLQ-C30 fatigue, nausea and vomiting, pain, dyspnea, insomnia and appetite loss scores, and the QLQ-STO22 dietary restrictions, pain, upper gastroesophageal, dry mouth and body image scores (HRs ranged from 1.27-1.85; Fig. 4). A reduction in the QLQ-C30 GHS score of 10 points increased the risk of ECOG PS deterioration by $51 \%$ (HR 1.5, 95\% CI 1.2-1.9).

\section{Discussion}

In patients with advanced refractory gastric cancer, for whom QoL has most likely already been diminished by disease progression and previous treatments, maintaining QoL is an important treatment goal. Indeed, QoL is included alongside OS in the European Society for Medical Oncology Magnitude of Clinical Benefit Scale (ESMO-MCBS), a proposed tool for measuring the potential clinical benefit of new anti-cancer therapies [15].

In this analysis of QoL data for TAGS, QoL was maintained from baseline for patients with pre-treated $(\geq 2$ regimens), histologically confirmed, non-resectable metastatic gastric adenocarcinoma, including adenocarcinoma of the gastroesophageal junction, who were receiving trifluridine/ tipiracil or placebo. As assessed by two widely used, validated, reliable and sensitive questionnaires (EORTC QLQC30 and QLQ-STO22), QoL for patients receiving trifluridine/tipiracil remained stable for all functional and symptom scales across treatment cycles $1-3$, and all scores except role functioning across cycles 4-6. There were no clinically relevant changes in the QLQ-C30 GHS score during treatment, nor were there any clinically relevant differences between treatment groups in this score over time. When death was considered as an event, there was a trend in favor of treatment with trifluridine/tipiracil. Although statistical significance was not achieved, patients treated with trifluridine/ tipiracil achieved numerically lower QoL changes than patients in the placebo group. Significant differences were observed in the sensitivity analysis including death or PD without previous deterioration in QoL as events. When both death and PD were considered as events, compared with placebo, patients treated with trifluridine/tipiracil achieved numerically lower changes in each QoL scores. By making the reasonable assumption that death and PD are associated with a decrease in QoL, time to deterioration (TTD) including deaths and PD as events might better capture the change in QoL and takes the missing data into account in a specific 
Fig. 3 Time to deterioration in EORTC QoL scores by $\geq 10$ points: sensitivity analyses including (a) death as an event and (b) disease progression and death as events. CI, confidence interval; EORTC, European Organization for Research and Treatment of Cancer; GHS, global health status; HR, hazard ratio; ITT, intention to treat; QLQ-C30, Quality of Life Questionnaire-Core 30; QLQ-STO22, Quality of Life Questionnaire-Gastric Cancer Module; QoL, quality of life. *Stratification factors were baseline Eastern Cooperative Oncology Group performance status (ECOG PS 0 vs 1) status, prior treatment with ramucirumab and region (Japan vs rest of World)

a

QoL Score [ $\left.{ }^{*}\right]$

QLQ-C30-GHS Physical functioning Role functioning

Emotional functioning Cognitive functioning

Social functioning

Fatigue

Nausea and vomiting

Pain

Dyspnea

Insomnia

Appetite loss

Constipation

Diarrhea

Financial difficulties

Dysphagia

Dietary restrictions

Pain QLQ-STO22

Upper gastroesophageal symptoms Anxiety

Dry mouth

Body image

Hair loss

Taste problems b

QoL Score [*]

QLQ-C30-GHS

Physical functioning

Role functioning

Emotional functioning

Cognitive functioning

Social functioning

Fatigue

Nausea and vomiting

Pain

Dyspnea

Insomnia

Appetite loss

Constipation

Diarrhea

Financial difficulties

Dysphagia

Dietary restrictions

Pain QLQ-STO22

Upper gastroesophageal symptoms

Anxiety

Dry mouth

Body image

Hair loss

Taste problems
HR $(95 \% \mathrm{Cl})$

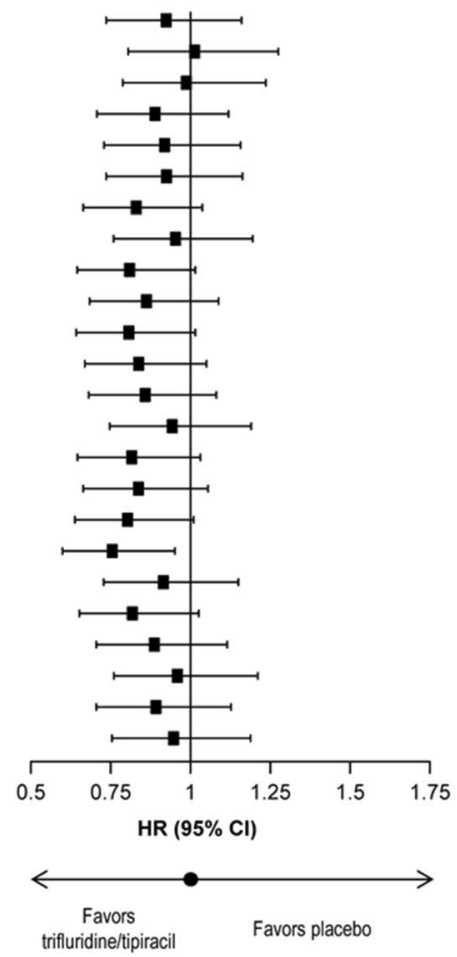

$0.92(0.74,1.16)$

$1.01(0.81,1.27)$

$0.99(0.79,1.24)$

$0.89(0.71,1.12)$

$0.92(0.73,1.16)$

$0.92(0.74,1.16)$

$0.83(0.66,1.04)$

$0.95(0.76,1.20)$

$0.81(0.65,1.01)$

$0.86(0.68,1.09)$

$0.81(0.64,1.02)$

$0.84(0.67,1.05)$

$0.86(0.68,1.08)$

$0.94(0.75,1.19)$

$0.82(0.65,1.03)$

$0.84(0.66,1.05)$

$0.80(0.64,1.01)$

$0.75(0.60,0.95)$

$0.92(0.73,1.15)$

$0.82(0.65,1.03)$

$0.89(0.71,1.11)$

$0.96(0.76,1.21)$

$0.89(0.71,1.13)$

$0.95(0.75,1.19)$

$\mathrm{HR}(95 \% \mathrm{Cl})$

$0.65(0.52,0.81)$

$0.71(0.57,0.89)$

$0.62(0.50,0.77)$

$0.66(0.53,0.82)$

$0.71(0.57,0.89)$

$0.62(0.50,0.77)$

$0.65(0.52,0.81)$

$0.75(0.60,0.93)$

$0.55(0.44,0.68)$

$0.65(0.52,0.81)$

$0.64(0.51,0.79)$

$0.63(0.51,0.78)$

$0.66(0.53,0.82)$

$0.72(0.58,0.90)$

$0.57(0.46,0.71)$

$0.62(0.50,0.77)$

$0.63(0.51,0.79)$

$0.59(0.48,0.74)$

$0.70(0.56,0.87)$

$0.59(0.47,0.73)$

$0.66(0.53,0.82)$

$0.75(0.60,0.93)$

$0.60(0.48,0.75)$

$0.70(0.57,0.87)$ 


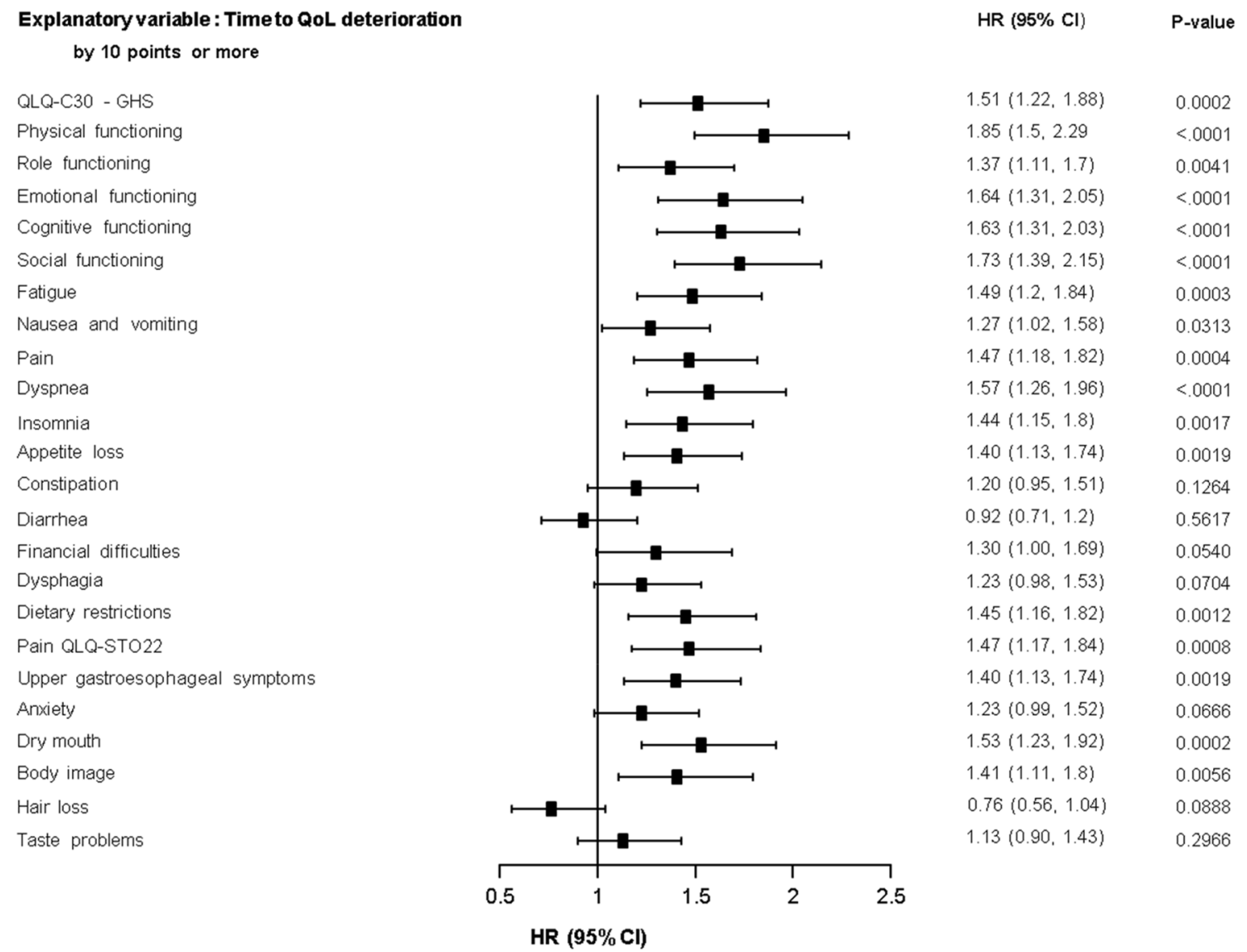

Fig. 4 Association between time to deterioration of ECOG PS to $\geq 2$ and time to deterioration of QoL score by $\geq 10$ points. CI, confidence interval; EORTC, European Organization for Research and Treatment of Cancer; GHS, global health status; HR, hazard ratio; ITT, inten- tion to treat; QLQ-C30, Quality of Life Questionnaire-Core 30; QLQSTO22, Quality of Life Questionnaire-Gastric Cancer Module; QoL, quality of life

include ATTRACTION-2 (third- or greater-line nivolumab vs placebo [16]), and JAVELIN Gastric 300 (third-line avelumab vs chemotherapy [17]); however, none of these trials reported a QoL analysis.

Limited QoL results were reported for a phase 3, randomized, double-blind study which compared third-line apatinib with placebo in Chinese patients $(n=267)$ with advanced gastric or gastroesophageal cancer [18]. Using the EORTC QLQ-C30 only, no significant between-group differences were observed at any measured time point for any of the QoL scores [18]. No data were given for the change in QoL from baseline, making it difficult to compare the QoL results with those from the current study. Based on the positive efficacy and acceptable safety results from this trial, apatinib was approved in China for patients with advanced gastric or gastroesophageal cancer who have progressed or relapsed after chemotherapy [19]. QoL endpoints were also assessed in the multinational ANGEL study, in which thirdline apatinib did not significantly improve OS compared with advanced gastric cancer who have received at least two previous lines of treatment). Key trials in similar patients 
with placebo [20, 21]; however, QoL results from this trial are not yet available.

A QoL sub-study of the phase 2 INTEGRATE study used the QLQ-C30, QLQ-STO22 and EuroQol-5D (EQ$5 \mathrm{D})$ questionnaires as well as the patient disease and treatment (PTDATA) form to measure QoL in patients $(n=142)$ with advanced gastric cancer receiving second- or third-line regorafenib or placebo [22]. To interpret results in a clinically meaningful way, both INTEGRATE and the current TAGS analyzed only results for which at least $10 \%$ of the original patient population completed QoL questionnaires. Using this approach, the TAGS data are robust enough to allow analysis for 3 treatment cycles $(\approx 12$ weeks) for placebo and 6 cycles $(\approx 24$ weeks) for trifluridine/tipiracil. Similarly, in INTEGRATE, the $10 \%$ cut-off point corresponded to week 8 for placebo and week 16 for regorafenib [22]. In both INTEGRATE and the current TAGS, baseline QLQ symptom scores were highest for anxiety, fatigue, body image, and appetite loss [22]. At week 4 in INTEGRATE, the mean diarrhea score was significantly higher for regorafenib than placebo; however, there were no other between-group differences in QoL scores at week 4 or 8 [22]. Although the QoL scores tended to worsen for both groups from baseline to week 8 in INTEGRATE, the rate of deterioration-free survival was significantly longer for regorafenib than placebo, leading the authors to conclude that regorafenib did not have an excessively negative effect on QoL [22]. When analyzing the prognostic value of baseline QoL scores for OS, adjusting for treatment allocation, OS was longer in patients with lower baseline scores for several symptoms (general pain, abdominal pain, appetite loss, constipation and eating restrictions), and higher baseline scores for physical functioning, role functioning and EQ-5D utility [22].

Although physicians intuitively associate disease progression with a deterioration in QoL, until recently this association had not been studied specifically for patients with gastric cancer [23]. To address this knowledge gap, a pooled analysis of QLQ-C30 data was conducted for two global phase 3 trials (RAINBOW and REGARD) in patients with gastric cancer receiving second-line therapy [23]. In this analysis, disease progression and deterioration in ECOG PS scores resulted in worse QoL scores [23]. Furthermore, changes from baseline to 6 weeks in global and functional scale QoL scores, as well as those in fatigue, pain and appetite loss scores, were predictive of changes in tumor status. As would be expected, a small change in the physical functioning score predicted an ECOG PS change [23]. The authors noted that these results emphasize the importance of disease control for the maintenance of QoL and suggested that QoL may be an additional useful tool for assessing tumor status in patients with non-measurable disease [23]. In TAGS, disease control was achieved by significantly more patients receiving trifluridine/tipiracil than placebo ( $44 \%$ vs $14.5 \%, p<0.0001$ ) [6]. In the current analysis, clinically relevant deteriorations in the QLQ-C30 GHS score and in the majority of other QoL scores were significantly associated with an increased risk of deterioration in ECOG PS to $\geq 2$.

Our study is not without limitations. The reduction in questionnaire completion rates over time meant it was not possible to assess the long-term effects of trifluridine/tipiracil treatment on QoL. Although the baseline questionnaire compliance rate was high, by treatment cycle 3 it was $\approx 85 \%$ for trifluridine/tipiracil and $70 \%$ for placebo; rates by cycle 6 had further decreased to $68 \%$ and $90 \%$, respectively. This limitation is common in cancer QoL studies and can be explained by patients discontinuing treatment. In the abovementioned trial of apatinib, questionnaire compliance rates at baseline and at the end of treatment cycles 2 and 3 were $100 \%, 60.8 \%$, and $34.7 \%$ for apatinib recipients and $100 \%$, $47.3 \%$ and $7.7 \%$, respectively, for placebo recipients [18]. In the INTEGRATE sub-study, there were no post-baseline QoL data available for $29 \%$ of regorafenib recipients and $41 \%$ of placebo recipients [22].

\section{Conclusions}

In this analysis of data from TAGS, QoL was maintained in patients with heavily pretreated, metastatic gastric cancer who received treatment with trifluridine/tipiracil, and there was a trend towards trifluridine/tipiracil reducing the risk of QoL deterioration compared with placebo. Together with previously published results showing that trifluridine/ tipiracil has a manageable safety profile and gives prolonged OS compared with placebo, these data support trifluridine/ tipiracil as a new treatment option in this difficult-to-treat patient population.

Acknowledgments We would like to thank Toni Dando of Springer Healthcare Communications who wrote the outline and first draft of this manuscript. This medical writing assistance was funded by Les Laboratoires Servier, France.

Author contributions Josep Tabernero, Maria Alsina, Kohei Shitara, Toshihiko Doi, Mikhail Dvorkin, Wasat Mansoor, Hendrik-Tobias Arkenau, Aliaksandr Prokharau, Michele Ghidini, Catia Faustino, Vera Gorbunova, Edvard Zhavrid, Kazuhiro Nishikawa, Takayuki Ando, Şuayib Yalçın, Eric Van Cutsem, and David H Ilson enrolled patients, discussed statistical analyses, participated in writing the manuscript, and read and approved the final draft before submission. Javier Sabater, Catherine Leger, and Nadia Amellal managed the logistical side of the substudy analyses, discussed statistical analyses, participated in writing the manuscript, and read and approved the final draft before submission. Donia Skanji performed statistical analyses, participated in writing the manuscript, and read and approved the final draft before submission. 


\section{Compliance with ethical standards}

Conflict of interest Josep Tabernero reports personal fees from Array Biopharma, AstraZeneca, Bayer, BeiGene, Boehringer Ingelheim, Chugai, Genentech, Inc., Genmab A/S, Halozyme, Imugene Limited, Inflection Biosciences Limited, Ipsen, Kura Oncology, Lilly, MSD, Menarini, Merck Serono, Merrimack, Merus, Molecular Partners, Novartis, Peptomyc, Pfizer, Pharmacyclics, ProteoDesign SL, Rafael Pharmaceuticals, F. Hoffmann-La Roche Ltd, Sanofi, SeaGen, Seattle Genetics, Servier, Symphogen, Taiho, VCN Biosciences, Biocartis, Foundation Medicine, HalioDX SAS and Roche Diagnostics. Maria Alsina reports personal financial interest in form of scientific consultancy for BMS, Lilly, MSD and Servier Honorarium for speaking issues from Amgen, BMS, Lilly, MSD, Roche and Servier Travel expenses partially covered by Amgen, Lilly and Roche. Kohei Shitara reports grants and personal fees from Astellas Pharma, Lilly, Ono Pharmaceutical, and MSD; personal fees from Bristol-Myers Squibb, Takeda, Pfizer, Novartis, Abbvie, and Yakult; grants from Dainippon Sumitomo Pharma, Daiichi Sankyo, Taiho Pharmaceutical, Chugai Pharma, and Medi Science. Toshihiko Doi reports grants from Lilly, Kyowa Hakko Kirin, MSD, Daiichi Sankyo, Amgen, Sumitomo Dainippon, Taiho, Novartis, Merck Serono, Astellas, Janssen, Boehringer Ingelheim, Takeda, Pfizer, Chugai Pharma, Celegene, Bristol-Myers Squibb, Abbvie, Quintiles, Bayer, and Eisai. Hendrik-Tobias Arkenau reports personal fees from Roche, Bicycle therapeutics, BioNTech, BeiGene. Michele Ghidini reports personal fees from Lilly, Servier for advisory boards. Catia Faustino reports personal fees from Servier, Merck Serono, Ipsen, Novartis, and Astellas. Kazuhiro Nishikawa reports personal fees from Chugai Pharma, Taiho Pharmaceutical, Yakult Honsha, Bristol-Myers Squibb Japan, EA Pharma, Lilly, and Ono Pharmaceutical; grants from Taiho Pharmaceutical, and Ono Pharmaceutical. Şuayib Yalçın reports honoraria from Roche, Pfizer, Amgen, Merck Serono, and Lilly. Eric Van Cutsem has received research funding from Amgen, Bayer, Boehringer Ingelheim, Celgene, Ipsen, Lilly, Merck, Merck KgA, Novartis, Roche, Sanofi, and Servier. Javier Sabater, Donia Skanji, Catherine Leger and Nadia Amellal are employees of Servier. David Ilson has served as a consultant/advisor for Amgen, Lilly, Roche, Genentech, Astra-Zeneca, Taiho, and Pieris and has received research funding from Bristol-Myers Squibb, Bayer, Taiho, and Amgen. Mikhail Dvorkin, Wasat Mansoor, Aliaksandr Prokharau, Vera Gorbunova, Edvard Zhavrid, and Takayuki Ando have no conflicts of interest to disclose.

Human rights statement All procedures followed were in accordance with the ethical standards of the responsible committee on human experimentation (institutional and national) and with the Helsinki Declaration of 1964 and later versions.

Informed consent Informed consent was obtained from all patients who were included in the study.

Open Access This article is licensed under a Creative Commons Attribution 4.0 International License, which permits use, sharing, adaptation, distribution and reproduction in any medium or format, as long as you give appropriate credit to the original author(s) and the source, provide a link to the Creative Commons licence, and indicate if changes were made. The images or other third party material in this article are included in the article's Creative Commons licence, unless indicated otherwise in a credit line to the material. If material is not included in the article's Creative Commons licence and your intended use is not permitted by statutory regulation or exceeds the permitted use, you will need to obtain permission directly from the copyright holder. To view a copy of this licence, visit http://creativecommons.org/licenses/by/4.0/.

\section{References}

1. The Global Cancer Observatory. Stomach cancer. http://gco. iarc.fr/today/data/factsheets/cancers/7-Stomach-fact-sheet.pdf. Accessed 28 Feb 2020.

2. Van Cutsem E, Sagaert X, Topal B, Haustermans K, Prenen H. Gastric cancer. Lancet. 2016;388:2654-64.

3. Berrino F, De Angelis R, Sant M, Rosso S, Bielska-Lasota M, Coebergh JW, et al. Survival for eight major cancers and all cancers combined for European adults diagnosed in 1995-99: results of the EUROCARE-4 study. Lancet Oncol. 2007;8:773-83.

4. Emura T, Suzuki N, Fujioka A, Ohshimo H, Fukushima M. Potentiation of the antitumor activity of alpha, alpha, alpha-trifluorothymidine by the co-administration of an inhibitor of thymidine phosphorylase at a suitable molar ratio in vivo. Int J Oncol. 2005;27:449-55.

5. Temmink OH, Emura T, de Bruin M, Fukushima M, Peters GJ. Therapeutic potential of the dual-targeted TAS-102 formulation in the treatment of gastrointestinal malignancies. Cancer Sci. 2007;98:779-89.

6. Shitara K, Doi T, Dvorkin M, Mansoor W, Arkenau HT, Prokharau A, et al. Trifluridine/tipiracil versus placebo in patients with heavily pretreated metastatic gastric cancer (TAGS): a randomised, double-blind, placebo-controlled, phase 3 trial. Lancet Oncol. 2018;19:1437-48.

7. US Food \& Drug Association. FDA approves Lonsurf for recurrent, metastatic gastric and gastroesophageal junction adenocarcinoma. http://www.fda.gov/Drugs/InformationOnDrugs/Appro vedDrugs/ucm632032.htm. Accessed 28 Feb 2020.

8. Taiho Pharma. Taiho Pharmaceutical obtains additional indication of gastric cancer in Japan for its anticancer agent LONSURF ${ }^{\circledR}$. https://www.taiho.co.jp/en/release/2019/20190822.html. Accessed 28 Feb 2020.

9. Taiho Pharma. Lonsurf ${ }^{\circledR}$ (trifluridine/tipiracil) receives marketing authorization in europe for previously treated metastatic gastric cancer https://www.taiho.co.jp/en/release/2019/20190906_1.html. Accessed 28 Feb 2020.

10. Vickery CW, Blazeby JM, Conroy T, Arraras J, Sezer O, Koller $\mathrm{M}$, et al. Development of an EORTC disease-specific quality of life module for use in patients with gastric cancer. Eur J Cancer. 2001;37:966-71.

11. Woo A, Fu T, Popovic M, Chow E, Cella D, Wong CS, et al. Comparison of the EORTC STO-22 and the FACT-Ga quality of life questionnaires for patients with gastric cancer. Ann Palliat Med. 2016;5:13-21.

12. Scott NW, Fayers PM, Aaronson NK, Bottomley A, de Graeff A, Groenvold M, et al. EORTC QLQ-C30 Reference Values. http:// www.eortc.org/app/uploads/sites/2/2018/02/reference_value s_manual2008.pdf. Accessed $28 \mathrm{Feb} 2020$.

13. Blazeby JM, Conroy T, Bottomley A, Vickery C, Arraras J, Sezer $\mathrm{O}$, et al. Clinical and psychometric validation of a questionnaire module, the EORTC QLQ-STO 22, to assess quality of life in patients with gastric cancer. Eur J Cancer. 2004;40:2260-8.

14. Osoba D, Rodrigues G, Myles J, Zee B, Pater J. Interpreting the significance of changes in health-related quality-of-life scores. J Clin Oncol. 1998;16:139-44.

15. Cherny NI, Dafni U, Bogaerts J, Latino NJ, Pentheroudakis G, Douillard JY, et al. ESMO-Magnitude of Clinical Benefit Scale version 1.1. Ann Oncol. 2017;28:2340-66.

16. Kang YK, Boku N, Satoh T, Ryu MH, Chao Y, Kato K, et al. Nivolumab in patients with advanced gastric or gastro-oesophageal junction cancer refractory to, or intolerant of, at least two previous chemotherapy regimens (ONO-4538-12, ATTRAC TION-2): a randomised, double-blind, placebo-controlled, phase 3 trial. Lancet. 2017;390:2461-71. 
17. Bang YJ, Ruiz EY, Van Cutsem E, Lee KW, Wyrwicz L, Schenker M, et al. Phase III, randomised trial of avelumab versus physician's choice of chemotherapy as third-line treatment of patients with advanced gastric or gastro-oesophageal junction cancer: primary analysis of JAVELIN Gastric 300. Ann Oncol. 2018;29:2052-60.

18. Jin L, Shukui Q, Jianming X, Jianping X, Changping W, Yuxian B, et al. Randomized, double-blind, placebo-controlled phase III trial of apatinib in patients with chemotherapy-refractory advanced or metastatic adenocarcinoma of the stomach or gastroesophageal junction. J Clin Oncol. 2016;34:1448-54.

19. Scott LJ. Apatinib: a review in advanced gastric cancer and other advanced cancers. Drugs. 2018;78:747-58.

20. LSK BioPharma. LSK BioPharma announces preliminary review of top-line results from ANGEL study. https://elevartherapeutics. com/2019/09/29/elevar-announces-results-from-the-angel-trial -a-study-of-monotherapy-rivoceranib-apatinib-in-late-stage-gastr ic-cancer-patients/. Accessed 28 Feb 2020.

21. Elevar Therapeutics (formely LSK BioPartners Inc). A prospective, randomized, double-blinded, placebo-controlled, multinational, multicenter, parallel-group, phase III study to evaluate the efficacy and safety of apatinib plus best supportive care (BSC) compared to placebo plus BSC in patients with advanced or metastatic gastric cancer [ClinicalTrials.gov record: NCT03042611]. http://clinicaltrials.gov/ct2/show/NCT03042611. Accessed 28 Feb 2020.

22. Martin AJ, Gibbs E, Sjoquist K, Pavlakis N, Simes J, Price T, et al. Health-related quality of life associated with regorafenib treatment in refractory advanced gastric adenocarcinoma. Gastric Cancer. 2018;21:473-80.

23. Chau I, Fuchs CS, Ohtsu A, Barzi A, Liepa AM, Cui ZL, et al. Association of quality of life with disease characteristics and treatment outcomes in patients with advanced gastric cancer: exploratory analysis of RAINBOW and REGARD phase III trials. Eur J Cancer. 2019;107:115-23.

Publisher's Note Springer Nature remains neutral with regard to jurisdictional claims in published maps and institutional affiliations.

\section{Affiliations}

\section{Josep Tabernero ${ }^{1}$ (D) Maria Alsina ${ }^{1} \cdot$ Kohei Shitara $^{2} \cdot{\text { Toshihiko } \text { Doi }^{2} \cdot \text { Mikhail Dvorkin }^{3} \cdot \text { Wasat Mansoor }}^{4} \cdot$ Hendrik-Tobias Arkenau ${ }^{5}$. Aliaksandr Prokharau ${ }^{6} \cdot$ Michele Ghidini $^{7}$. Catia Faustino ${ }^{8}$. Vera Gorbunova ${ }^{9}$. Edvard Zhavrid ${ }^{10} \cdot$ Kazuhiro Nishikawa $^{11} \cdot$ Takayuki Ando $^{12}$ - Şuayib Yalçın ${ }^{13}$. Eric Van Cutsem ${ }^{14}$. Javier Sabater ${ }^{15}$. Donia Skanji ${ }^{16}$. Catherine Leger ${ }^{16}$. Nadia Amellal ${ }^{16}$. David H. Ilson ${ }^{17}$}

1 Vall d'Hebron University Hospital and Institute of Oncology (VHIO), UVic-UCC, IOB-Quiron, Barcelona, Spain

2 National Cancer Center Hospital East, Chiba, Japan

3 Omsk Regional Clinical Centre of Oncology, Omsk, Russia

4 The Christie NHS Foundation Trust, Manchester, UK

5 Sarah Cannon Research Institute, London, UK

6 Minsk City Clinical Oncology Dispensary, Minsk, Belarus

7 Azienda Ospedaliera di Cremona, Cremona, Italy

8 Instituto Português de Oncologia do Porto Francisco Gentil, Porto, Portugal

9 N.N. Blokhin Russian Cancer Research Center, Moscow, Russia
10 Alexandrov National Cancer Centre of Belarus, Minsk, Belarus

11 Osaka National Hospital, Osaka, Japan

12 University of Toyama, Toyama, Japan

13 Hacettepe University, Ankara, Turkey

14 University Hospitals and KU Leuven, Leuven, Belgium

15 Market Access Department, Servier, Suresnes, France

16 Institut de Recherches Internationales Servier, Suresnes, France

17 Memorial Sloan Kettering Cancer Center, New York, NY, USA 